\title{
Priapism and glucose-6-phosphate dehydrogenase deficiency: An underestimated correlation?
}

\author{
Aldo Franco De Rose ${ }^{1}$, Guglielmo Mantica ${ }^{1}$, Mattia Tosi ${ }^{1}$, Giulio Bovio ${ }^{2}$, Carlo Terrone ${ }^{1}$ \\ ${ }^{1}$ Department of Urology, IRCCS San Martino Hospital, University of Genova, Genova, Italy; \\ ${ }^{2}$ Department of Radiology, IRCCS San Martino Hospital, University of Genova, Genova, Italy.
}

\begin{abstract}
Summary
Priapism is a rare clinical condition characterized by a persistent erection unrelated to sexual excitement. Often the etiology is idiopathic. Three cases of priapism in glucose-6-phosphate dehydrogenase (G6PD) deficiency patients have been described in literature. We present the case of a 39-year-old man with glucose-6-phosphate dehydrogenase deficiency, who reached out to our department for the arising of a non-ischemic priapism without arteriolacunar fistula. We suggest that the glucose-6-phosphate dehydrogenase deficiency could be an underestimated risk factor for priapism.
\end{abstract}

KEY WORDS: Non-ischemic; Glucose-6-phosphate dehydrogenase (G6PD) deficiency.

Submitted 14 May 2016; Accepted 30 May 2016

\section{INTRODUCTION}

There are three types of priapism with different pathophysiological causes: the ischemic priapism (or "low flow"), which is a dolorous and prolonged erection with a tissue ischemia, the non-ischemic (or "high flow") priapism, which is usually due to a perineal injury with the formation of an arteriolacunar fistula, and the stuttering (intermittent) priapism, which is a recurrent form of ischemic priapism in which unwanted painful erections occur repeatedly with intervening periods of detumescence. All types of priapisms can lead to an erectile dysfunction (1), not only the ischemic one. For that reason, it is important to treat it in time and in correct way, but also to identify the possible risk factors. Three cases of priapism in patients with G6PD deficiency have been described in literature (2). We report an unique case of "high flow" priapism without fistula in a patient with G6PD deficiency.

\section{Case presentation}

In October 2015, a 39-year-old man reached to our observation for an intermittent, painless erection, everyday, for about one month. He reported erections lasting about 4-6 hours during the first days of the month. These erections stopped in the following days, then he had another erection lasting more than 17 hours, which obliged him to go to the emergency department. He reported a normal and spontaneous erectile function before the arising of the disease. He denied having any gender of trauma and he admitted to have a G6PD deficiency, documented by laboratory tests. He denied having taken any drugs, phosphodiesterase type 5 (PDE5) inhibitors, intracavernosal agents or any other pharmacological agents. He denied any other risk factor for priapism. At clinical examination he presented a painless erection. Systemic hemoglobin was $13.6 \mathrm{~g} / \mathrm{dL}$.

Intracorporal blood resulted as arterial at gas analysis $\left(\mathrm{PO}_{2}=76 \mathrm{mmHg} ; \mathrm{PCO}_{2}=44.5 \mathrm{mmHg} ; \mathrm{pH}=7.35\right)$. The Color Doppler ultrasonography didn't point out any arterocavernosa fistula. After a period of observation in which there wasn't the resolution of the priapism, we decided to submit him to a pelvic arteriography (Figure 1).

Panoramic arteriography of the aorto-iliac and selective of internal pudendal artery showed a bilateral increase of the arterial level of the corpora cavernosa, higher in the left one, without a fistula. We performed a temporary embolization with Spongostan ${ }^{T M}$ of the distal left internal pudendal artery. A complete detumescence was obtained and the next arteriography confirmed the procedure was a success. The patient was released.

After 2 weeks of a state of good health, he presented a new episode of prolonged erection, after which he returned to the emergency department of our Institute.

\section{Figure 1.}

Pelvic arteriography showing a bilateral increase of the arterial level of the corpora cavernosa, higher in the left one, without a fistula.

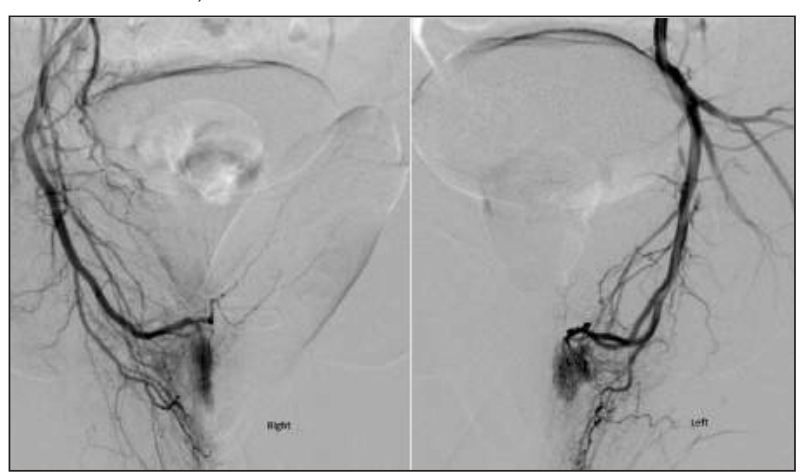


This time the erection was higher and mildly painful. He still denied having taken drugs or medications. We performed a gas analysis $\left(\mathrm{pO}_{2}=21.3 \mathrm{mmHg} ; \mathrm{pCO}_{2}=56.4\right.$; mmHg; $\mathrm{pH}=7.34$ ). We carried out an evacuation of blood from the cavernosa corpora through transglandular cannula and injection of Etilefrine which lead to a detumescence. After an observation time of 24 hours without erections, we decided to discharge the patient from our department. Three months later he affirmed to have preserved a sufficient erectile function (IIEF-5: 18).

\section{Conclusions}

The number of priapisms, classified as idiopathic, both ischemic and not-ischemic, continues to be high. Among the clinically significant enzymatic defects, G6PD deficiency is the most common (3), with hundreds and millions of affected people. With the proliferation of the G6PD deficiency, especially in some countries, we suggest it could be a casual factor or a co-factor, responsible for some cases of priapism classified as idiopathic. Therefore, now we consider the G6PD deficiency as a underestimated risk factor for the emergence of priapism.

\section{Discussion}

Priapism can be associated with some hematologic, coagulative and enzymatic disorders (4-5) such as leukemia and sickle cell anemia. Hematologic diseases are usually associated with a "low flow" priapism, but some associations have also been reported between "high flow" priapism and sickle cell anemia (6). Both type of priapism are also associated with Fabry disease $(7,8)$. Nowadays, few cases of priapism, related to a G6PD deficiency, are present in literature, even if the correlation between the two disorders has been discovered $(2,9,10)$. The cases, which have emerged until now, showed a link between the G6PD deficiency and the "low flow" priapism. In our case, the patient initially showed a "high flow" priapism, without the presence of other risk factors for the disor- der. The interesting thing was the absence of fistula at the arteriography, but the presence of an increase of the blood flow in the corpora cavernosa. Although the arrangements are unknown, the G6PD deficiency could be responsible for that increase of blood flow and it could lead to a not-ischemic priapism other than an ischemic one, as it's already documented and assumed for the other enzymatic and hematologic defects (6-8).

\section{REFERENCES}

1. Zacharakis E, Ralph David J, Walkden M, et al. Distal corpus cavernosum fibrosis and erectile dysfunction secondary to nonischaemic priapism. Arch It Urol Androl. 2015; 87:258-9.

2. Morrison BF, Thompson EB, Shah SD. Ischaemic priapism and glucose-6-phosphate dehydrogenase deficiency: a mechanism of increased oxidative stress? West Indian Med J. 2014; 63:658-60.

3. WHO Working Group. Glucose-6-phosphate dehydrogenase deficiency. Bull World Health Organ. 1989; 67:601-11.

4. Kalathia J, Agrawal S, SudirChipde S, Agrawal R. Homocysteinemia: a rare cause of priapism. Urol Ann 2016; 8:118-21.

5. Morrison BF, Burnett AL. Priapism in hematological and coagulative disorders: an update. Nat Rev Urol. 2011; 8:223-230.

6. Ramos CE, Park JS, Ritchey ML, Benson GS. High flow priapism associated with sickle cell disease. J Urol. 1995; 153:1619-1621.

7. Foda MM, Mahmood K, Rasuli P, et al. High-flow priapism associated with Fabry's disease in a child: a case report and review of the literature. Urology. 1996; 48:949-952.

8. Backenroth R, Landau EH, Goren M, Raas-Rothschild A. Fabry disease and G6PD in three family members with priapism: is the nitric oxide pathway to blame? J Sex Med. 2010; 7:1588-1591.

9. Burnett AL, Bivalacqua TJ. Glucose-6-phosphate dehydrogenase deficiency: an etiology for idiopathic priapism? J Sex Med. 2008; 5:237-240.

10. Finley DS. Glucose-6-phosphate dehydrogenase deficiency associated stuttering priapism: report of a case. J Sex Med. 2008; 5:2963-2966.

\section{Correspondence}

Aldo Franco De Rose, MD

aldofrancoderose@gmail.com

Guglielmo Mantica, MD (Corresponding Author)

guglielmo.mantica@gmail.com

3383699431

Mattia Tosi, MD

matti.grifone@hotmail.it

Carlo Terrone, MD

Department of Urology, IRCCS San Martino Hospital, University of Genova

Largo Rosanna Benzi 10 - 16131, Genova, Italy

Giulio Bovio, MD

giulio.bovio@hsanmartino.it

Department of Radiology, IRCCS San Martino Hospital, University of Genova

Largo Rosanna Benzi 10 - 16131, Genova, Italy 